\title{
BLENDED LEARNING AND LOCALNESS: THE MEANS AND THE END
}

\author{
A. Frank Mayadas \\ Program Officer \\ The Alfred P. Sloan Foundation
}

Anthony G. Picciano

Professor, School of Education, Hunter College

Doctoral Programs in Urban Education, and Interactive Pedagogy and Technology

City University of New York Graduate Center

\begin{abstract}
Blended learning can be seen as the means to achieving a greater sense of "localness" on the part of colleges and universities. Blended learning has been evolving for several years and while definitions vary from one institution to another, it is defined in this paper essentially as a combination of face-to-face and online learning. Localness is a term used at the Alfred P. Sloan Foundation as part of a new funding initiative to support academic programs designed to strengthen a college or university connection to its core constituencies. The purpose of this paper is to explore the relationship of blended learning and "localness".
\end{abstract}

\section{KEYWORDS}

Distance Learning, Online Learning, Blended Learning, Hybrid Learning, Localness, Access, Marketing, Branding

\section{INTRODUCTION}

At the Sloan Consortium Summer Workshop held in Baltimore in 2006, a panel discussion was organized entitled, Localness and Blended Approaches to ALN. Seven presentations were made: two were designed to introduce the topic and five described case studies of blended learning applied to localness initiatives. The Alfred P. Sloan Foundation has been funding activities in ALN (asynchronous learning networks) since the early 1990s as part of its Anytime, Anyplace Learning initiative. While the term ALN is often used interchangeably with online learning, in its purest form, it referred specifically to asynchronous, instructor-led, highly-interactive, cohort-based online learning environments. The Alfred P. Sloan Foundation's interest was to use ALN as a way to expand access to higher education opportunities for students who were geographically distant or time-constrained. Blended learning is a more recent phenomenon and refers to the blending or mixing of face-to-face and online learning in an academic program or course [1]. The Alfred P. Sloan Foundation sponsored two workshops (2004 and 2005) hosted by the University of Illinois, Chicago, that focused on definition, research, and institutional practices of blended learning. In 2006, a third workshop was funded which added the concept of "localness' to the blended learning theme. Localness referred to a new initiative of The Alfred P. Sloan Foundation in support of academic programs designed to strengthen and enhance an institution's connections to its core constituencies. A fundamental aspect of the panel discussion on Localness and Blended Approaches to $A L N$, was to continue the work started in Chicago and to explore further blended learning as a means for 
higher education institutions to expand their connections to their local or core communities.

The purpose of this paper is to explore the relationship of blended learning and "localness". Models of programs are presented to demonstrate this relationship.

\section{DEFINITIONS}

\section{A. Blended Learning}

Blended learning, also known as hybrid and mixed-mode learning, is not one thing. It comes in many shapes, flavors, and colors. In one course, blended learning may be the enhancement of the traditional lecture with electronic instructor notes, additional readings, and images of charts, graphs, or other handouts. In another course, online learning may be combined with face-to-face instruction so that it meets two hours per week in a classroom with the third hour consisting of an online threaded discussion [1]. As noted by Gary Miller, Associate Vice President for Outreach and former Executive Director of The World Campus, the Pennsylvania State University recently went through a lengthy process that resulted in the definition of five variations of "blended learning" environments [2].

In the broadest sense, blended learning can be defined or conceptualized as any combination of a wide variety of technology/media integrated into conventional, face-to-face classroom activities. However, the blended learning variation examined in this paper focuses on an online component with some replacement of seat time in the conventional classroom. This definition was discussed extensively at the 2004 and 2005 Sloan-C Workshops on Blended Learning held in Chicago. The two core elements (online and faceto-face instruction) of this definition were deemed critical to the definition. This definition eliminates certain forms of stand-alone media such as videotape, CD-ROM, or DVD that might be used solely in a face-to-face course. It does not eliminate these media if used in a course that has both an online and a face-to-face component.

At the 2005 Sloan-C Workshop on Blended Learning, the following was adopted by the participants and will serve as the definition of blended learning for this paper:

1. Courses that integrate online with traditional face-to-face class activities in a planned, pedagogically valuable manner;

2. A portion (institutionally defined) of face-to-face time is replaced by online activity [3].

It is understood that this definition incorporates a number of online learning techniques (e.g., webcasting, discussion boards, text messaging) delivered synchronously or asynchronously. However, in keeping with the original Alfred P. Sloan Foundation initiatives in ALN stated earlier, the Sloan Consortium recommends that these techniques continue to be instructor-led and highly interactive and not simply electronic delivery of course content.

\section{B. Localness}

The application of localness to ALN and blended learning was first articulated in an unpublished paper distributed by the Alfred P. Sloan Foundation [4] focusing on connections of higher education institutions to their local communities and/or radii of influence. Most traditional, non-profit institutions with large commuter, non-residential and part-time student populations are well-known and trusted within their localities. When online learning burst into the academic consciousness in the mid-90's there was a rush by many of these institutions to downplay their locality, and to emphasize their role in meeting the needs 
Blended Learning and Localness: The Means and the End

of all kinds of geography-independent and global student populations. However, many of these same institutions eventually came to realize that many of their local and in some cases even their residential student populations were as interested in enrolling in online learning courses as were students living afar. The institutions are known in their local regions; that's not the issue. What is not always known is that they are offering a "quality" online or blended product. To provide a multiplicity of options for all students, educational institutions should consider strengthening their positions within their local regions by expanding their ALN and blended programs and by then making it well-known that such programs exist. A strong ALN or blended effort might also be used to permit institutions to extend and expand their effective core constituent bases. This concept is called localness.

\section{MODEL PROGRAMS}

The typical example of localness would be a community college or public university that expands or adds fully online or blended learning programs to allow greater access to higher education for its local core student populations. For example, the University of Massachusetts offers staff development and other outreach programs to local public schools. In order to reduce some of the travel time for education professionals who combine education, careers, and family responsibilities into incredibly busy days, portions of these outreach programs are also available in an online mode. Similar blended approaches could also be taken with any of the local professional areas such as health science, nursing, business, social work, or engineering.

Another example is the City University of New York's recently implemented Online BA program in Communication and Culture which is specifically designed for it own students who withdrew in good academic standing from any of the traditional programs in its colleges. CUNY like many large public university systems casts a wide net of access to higher education but also has a high drop-out rate especially among first-year students. However, the vast majority of these students drop-out because of financial, family, or career responsibilities and not because they are unable to do academic work. The convenience of an online degree program might help these students balance their personal lives with their higher education aspirations.

Since first being introduced in early 2006, the concept of localness has been expanding to include connections with constituencies that are not necessarily geographically local. Higher education institutions that have developed constituencies that exist beyond a locality or subsequently grow beyond a locality can also be models of localness. For example, Pace University has developed a number of certificate programs to develop and maintain skills for workers in the telecommunications industry. What originally started as the University's connection with a local business and a local trade union while still in existence has expanded into a national constituency of telecommunications businesses and a parent trade unions. ALN and blended learning are being used to reduce the need for employees and workers to travel extensively from different parts of the country to take advantage of Pace University's program.

Babson College, located in Needham, Massachusetts, has been experimenting with a multi-tier localness strategy for graduate business education. By "localness" Babson means the reach of the college within a finite geographic area not necessarily in Needham or the Boston area. Babson is committed to a blended MBA, which has served students living in relatively close proximity to the college. However, Babson is also seeking to extend its reach in geographically distant locations through what it terms a geo-local strategy. At the time of this writing, the college was planning to offer a blended MBA program specifically in Portland, Oregon where it has already established ties to several local businesses. 
These models exemplify ways in which ALN and blended learning can be used to establish and strengthen the connection of higher education institutions to their core constituencies. In these examples, the goal or objective was to strengthen the connection (localness) and blended learning was the vehicle or means to facilitate the connection.

Before concluding this section, it is also important to mention that one aspect of these localness initiatives is to confirm in the minds of constituents the importance of the educational services provided by these traditional colleges and universities. Marketing, branding, and advertisement of these services are critical to strengthening the connections with their core constituents, whether they are traditional students, businesses, public agencies, or whole industries. The vehicle of blended learning likewise is one important way for colleges and universities to demonstrate that they are sensitive to and willing to address the personal, financial, and other needs of these constituents.

\title{
IV. CONCLUDING COMMENTS
}

The connection of localness to blended learning presented in this paper evolved from discussions and presentations at the 2006 Sloan-C Summer Workshop held in Baltimore; the 2004 and 2005 Sloan-C Workshops on Blended Learning, and the 2006 Sloan-C Workshop on Blended Learning, Localness, and Outreach held in Chicago. The workshop participants constituted a group of administrators and faculty experienced in looking at issues of online learning. Their insights were expressed formally during workshop sessions as well as informally at social gatherings. This paper attempts to organize some of these insights and to provide clarity to the definitions and concepts of localness and blended learning as well as emphasize the importance of their relationship to each other. It was the opinion of these participants that blended learning is expanding rapidly throughout higher education and is evolving into one of the most important formats for delivering instruction [5]. When used as a means to achieve stronger ties with students and other constituents within an institution's realm, it has the potential of significantly extending and enriching educational opportunities.

\section{ACKNOWLEDGEMENTS}

The authors of this paper are grateful to colleagues who participated on the panel discussion, Localness and Blended Approaches to ALN, at the 2006 Sloan-C Summer Workshop, specifically:

\author{
Jacquie Moloney, University of Massachusetts, Lowell \\ Steve Laster, Babson College \\ George Otte, City University of New York \\ Bob Ubell, Stevens Institute of Technology \\ David Sachs, Pace University
}

The authors also thank Elaine Bowden for reviewing earlier drafts of this article and offering her suggestions.

\section{REFERENCES}

1. Picciano, A. G. Blended Learning: Implications for growth and access. Journal of Asynchronous Learning Networks 10(3): 2006. http://www.sloan-c.org/publications/jaln/v10n3/index member.asp. 
Blended Learning and Localness: The Means and the End

2. Miller, G. Blended learning and Sloan-C. Posting to the Official Website of the 2005 Sloan-C Summer Workshop held in Victoria, British Columbia, June 30, 2005.

3. Laster, S., G. Otte, A. G. Picciano, and S. Sorg. Redefining blended learning. Presentation at the 2005 Sloan-C Workshop on Blended Learning, Chicago, IL, April 18, 2005.

4. Mayadas, A. G. Blended, ALN, and localness initiative. Unpublished paper. New York: The Alfred P. Sloan Foundation, 2006.

5. Picciano, A. G. and C. D. Dzuiban (Eds.). Blended Learning: Research Perspectives. Needham. MA: Sloan-C, 2007.

\section{ABOUT THE AUTHORS}

Frank Mayadas is a Program Officer for The Alfred P. Sloan Foundation. Prior to the Sloan Foundation, Dr. Mayadas spent 27 years at the IBM Corporation where he was Vice President, Research Division, Technical Plans and Controls from 1991 to 1992; Vice President, Technology and Solutions Development, Application Solutions Line of Business, from 1989 to 1991; General Manager, University and College Systems, IBM Personal Systems Line of Business, from 1988 to 1989; Secretary of IBM's Corporate Management Board and the IBM Management Committee, from 1987 to 1988; and the IBM Management Committee, from 1987 to 1988; IBM Research Division Vice President and Director, Almaden Research Center, San Jose, California from 1983 to 1987; and an IBM Research Division Director, Technical Planning and Controls, from 1981 to 1983. At the Sloan Foundation, Dr. Mayadas is involved in a number of areas: online education, globalization of industries, industry studies, and career choice in technical fields. He started the Sloan online learning program in 1993. This program (known as Anytime/Anyplace Learning) has had a profound impact in moving the academic field forward. Academic partners in the effort comprise the Sloan Consortium or Sloan-C (see www.sloan-c.org). A large number of disciplines are covered by the consortium, ranging across humanities, engineering, business, and social-, life- and physical- sciences. Over 900 full programs (Certificate and Degree) are offered by consortium members.

Anthony G. Picciano is a professor in the Educational Leadership program at Hunter College. He is also a professor in the Ph.D. Program in Urban Education and the program in Interactive Pedagogy and Technology at the City University of New York Graduate Center. His teaching specializations include educational technology, contemporary issues in education, educational policy, organization theory, and research methods. He is the author of seven books including Educational Leadership and Planning for Technology, 4th Ed. (Pearson, 2005), Data-Driven Decision Making for Effective School Leadership (Pearson, 2006), Educational Research Primer (Continuum, 2004), and Distance Learning: Making Connections across Virtual Space and Time (Merrill/Prentice Hall, 2001). He has received a number of grants and awards from the National Science Foundation, the Alfred P. Sloan Foundation, the US Department of Education, and IBM. He currently serves on the Board of Directors of the Sloan Consortium of Colleges and Universities. His articles and reviews have appeared in journals such as The Teachers College Record, The Urban Review, Journal of Asynchronous Learning Networks, Journal of Thought, Journal of Educational Multimedia and Hypermedia, Computers in the Schools, Equity and Choice, and EDUCOM Review. 